\title{
Hunting the Chameleon: Structural Conformations of the Intrinsically Disordered Protein Alpha-Synuclein
}

\author{
Malte Drescher, ${ }^{*[a]}$ Martina Huber, ${ }^{[b]}$ and Vinod Subramaniam ${ }^{[a, c]}$
}

\section{Introduction}

\section{Alpha-synuclein}

The human $\alpha$-synuclein protein (ASYN) plays a central role in the etiology of Parkinson's disease, ${ }^{[1]}$ and forms fibrillar aggregates that are found in Lewy bodies and Lewy neurites in the brain, structures which are the hallmark of the disease. ${ }^{[2-4]}$ Three point mutations (A30P, A53T, and E46K) ${ }^{1}$ are associated with early-onset Parkinson's disease. ${ }^{[5-7]}$ Modifications such as phosphorylation $^{2}$ of the serine residue at position 129, and truncations of the protein, are reported to play an important role in the toxicity of ASYN. ${ }^{[8-11]}$

ASYN displays remarkable structural versatility: it has long been considered an intrinsically disordered or "natively unfolded" protein at physiological conditions, but can readily adopt $\beta$-sheet structure in aggregates or $\alpha$-helical structure when bound to lipids. ${ }^{[12-15]}$ Very recent reports suggest that at physiological conditions in vivo, ASYN adopts a helical tetrameric structure, ${ }^{[16-17]}$ although this observation remains controversial. ${ }^{[18]}$ The intriguing reports about the ASYN tetramer could imply that these structures are yet another face of the chameleon-like nature of the protein in vivo. The notion of $\alpha$-synuclein as a protein chameleon was introduced in 2003 by Uversky, ${ }^{[19]}$ and the ensuing years have only served to reinforce this view of the protein. The function of ASYN is unknown, but it is thought to involve lipid-binding in vesicles and synaptic membranes. ${ }^{[20-26]}$ The intrinsically disordered nature of the protein renders it intractable to standard high-resolution structural biology methods, making single-molecule Förster resonance energy transfer (FRET) and spectroscopic approaches that yield distance constraints invaluable in establishing structural parameters for the protein. ${ }^{[27-35]}$

In general for amyloid diseases, early aggregation intermediates are suspected of playing a key role in cell damage, although the structures and mechanisms of action of these early intermediates are unknown. ${ }^{[36]}$ There is evidence that amyloid toxicity may be caused by membrane permeabilization by pore-like early intermediates leading to disruption of calcium homeostasis and cell-death; ${ }^{[37-44]}$ such pores have been detect-

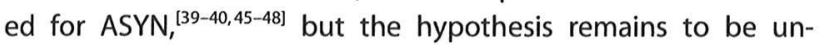
equivocally verified. Thus, it is very likely that the structural di-

\footnotetext{
${ }^{0}$ Phosphorylation is the addition of a phosphate $\left(\mathrm{PO}_{4}{ }^{3}\right)$ group to a protein or other organic molecule. Protein phosphorylation plays an important role in the regulation of cellular processes, including signal transduction.
}

versity of ASYN is a key element in the pathology of the synucleinopathies. ${ }^{[49]}$

\section{Labeling Approaches and Methods for Dis- tance Measurements in the Nanometer Range}

\section{Site-directed labeling}

Site-directed labeling is an essential enabling methodology for both fluorescence and electron paramagnetic resonance (EPR) measurements, and facilitates the directed coupling of a suitable fluorophore or spin label on the protein of interest. In general, a cysteine residue is introduced at specific amino-acid positions, to which a fluorescent or spin label is covalently attached using thiol chemistry. Because ASYN has no intrinsic cysteine residues, singly and doubly labeled ASYN derivatives can be generated by site-directed mutagenesis to introduce cysteines at any position in the primary sequence, followed by labeling. Alternatively, site-directed mutagenesis can be used to introduce an intrinsically fluorescent amino acid, such as tryptophan (Trp), at the desired position. Wild-type ASYN has four weakly fluorescent tyrosine residues, and no Trp residues, which makes fluorescence studies of Trp substitution mutants of ASYN a valuable approach to study ASYN conformations, conformational dynamics, and membrane interactions. ${ }^{[50-60]}$

In site-directed spin labeling electron paramagnetic resonance (SDSL-EPR) spectroscopy, nitroxides are usually used as spin-labels. ${ }^{[61-62]}$ Nitroxides are stable free radicals of the general form $\cdot O-N R^{1} R^{2}$ where the unpaired electron required for EPR detection is (de)localized on the $\mathrm{N} \mathrm{O}$ bond. Since many biological macromolecules (including ASYN) are diamagnetic, the nitroxide resonance is most often the only signal in the EPR spectrum. The most common spin labeling strategy for pro-

[a] Dr. M. Drescher, Prof. Dr. V. Subramaniam

Department of Chemistry, Konstanz Research School Chemical Biology and Zukunftskolleg, University of Konstanz

78457 Konstanz (Germany)

Email:malte.drescher@uni konstanz.de

[b] Dr. M. Huber

Department of Molecular Physics, Leiden University 2300RA Leiden (The Netherlands)

[c] Prof. Dr. V. Subramaniam

Nanobiophysics, MESA+ Institute for Nanotechnology

and MIRA Institute for Biomedical Technology and Technical Medicine University of Twente

7500 AE Enschede (The Netherlands) 


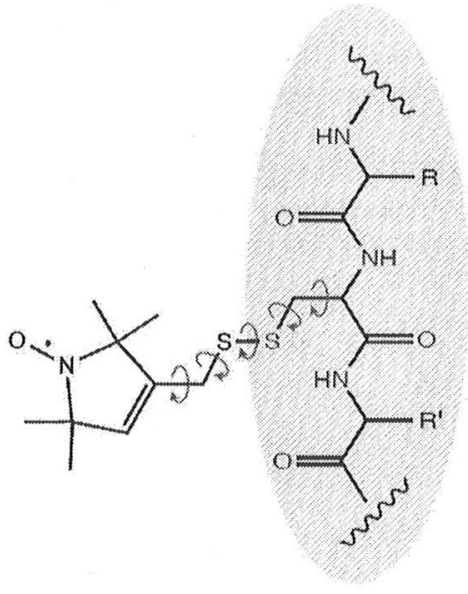

Scheme 1. Structure of MTSL reacted with a cysteine residue within an amino acid sequence (shaded). The rotational degrees of freedom caused by participating single bonds are indicated. Taken from ref. [84] with permis sion. Copyright: Springer Verlag, 2011.

teins uses cysteine substitution mutagenesis followed by modification of the unique sulfhydryl group with specific labeling reagents, for example, (1-oxyl-2,2,5,5-tetramethylpyrroline-3methyl)-methanethiosulfonate (MTSL, Scheme 1). ${ }^{[63]}$ MTSL has become the spin label of choice in SDSL studies, particularly because it is tolerated surprisingly well at the vast majority of sites at which it has been introduced into many different proteins. It is small and has been shown to have minimal effects on protein structures. ${ }^{[64-65]}$

\section{Förster resonance energy transfer (FRET)}

FRET is a nonradiative transfer of the excitation energy from a donor fluorophore to an acceptor chromophore. ${ }^{[66-67]}$ FRET involves a distance-dependent interaction between the emission and absorption transition dipole moments of the donor and acceptor molecules respectively. The rate of energy transfer depends on the spectral overlap of the donor emission and acceptor absorbance spectra, the donor fluorescence quantum yield, the relative orientations of the donor and acceptor transition dipole moments, and the distance between donor and acceptor molecules. The energy transfer rate, $k_{\mathrm{E},}$ is given by [Eq. (1)]:

$k_{E T}=\frac{1}{\tau_{d}}\left(\frac{R_{0}}{r}\right)^{6}$

where $\tau_{\mathrm{d}}$ is the lifetime of the donor fluorophore in the $a b-$ sence of an acceptor, and $r$ is the donor-acceptor distance. The Förster radius $R_{0}$ (typically 2 to $9 \mathrm{~nm}$ ) characterizes a given donor/acceptor pair, and is defined as the distance at which the efficiency of resonance energy transfer is $50 \%$. The resonance energy transfer provides an additional deactivation pathway for the excited fluorophore and results in reduced excited state lifetime of the donor fluorophore. Förster radii of commonly used donor/acceptor pairs are comparable to the size of biological macromolecules, resulting in FRET being extensively used as a "spectroscopic ruler" for measuring distances between sites on proteins. In particular, ensemble ${ }^{[51,54,59,60]}$ and single-molecule FRET ${ }^{[34,35,68-71]}$ approaches have been used for measuring conformational fluctuations in ASYN. Note that the magnitude of $k_{E T}$ can be determined from the efficiency of energy transfer, $E_{T}$ using the relation [Eq. (2)]:

$k_{E T}=\frac{1}{\tau_{d}}\left(\frac{E_{T}}{1} E_{T}\right)$

$E_{\mathrm{T}}$ can be determined experimentally by measuring the decrease in the intensity or the lifetime of the donor in the presence of the acceptor [Eq. (3)],

$E_{T}=1 \quad \frac{F}{F_{d}}=1 \quad \frac{\tau}{\tau_{d}}$

\section{Electron paramagnetic resonance spectroscopy}

Since the conformation of ASYN sensitively depends on the environment, corresponding structural details are notoriously difficult to unravel. The determination of unique high-resolution structures remains an elusive target for ASYN, but SDSL-EPR offers a powerful methodology to get structural insights into ASYN. Advantages of SDSL-EPR include high sensitivity and virtually no limitation on the size of the object under study. Since unlabeled diamagnetic molecules are EPR silent, distance constraints can be obtained background free in (frozen) solution. While FRET requires two different, relatively large, chromophores, which must be chosen according to the expected distance, EPR distance measurements can be performed using two identical, much smaller, nitroxide labels featuring minimal perturbations of the system under investigation and which are precise over a broad range of distances. ${ }^{[72-74]}$ Different experimental approaches can access distances between paramagnetic centers in the range between 1 and $10 \mathrm{~nm} ;:^{[63,72,75-82]}$ for recent reviews see refs. [83] and [84].

Distances between nitroxide spin labels below $2 \mathrm{~nm}$ can be derived by continuous wave (CW) EPR. The spectrum of interacting spins can be treated as the convolution of the non-interacting spectrum with a dipolar broadening function from which the distance can be derived. Pulsed methods ${ }^{[85]}$ increase the range of distance sensitivity; double electron-electron resonance (DEER, an acronym which is used synonymously with PELDOR (pulsed electron double resonance)) is the most widely applied in the field. ${ }^{[72,77,86-87]}$ DEER is an attractive method for its unsurpassed accuracy and its ability to extract distance distributions ${ }^{[88-94]}$ allowing for analyzing flexible structures or coexisting conformations. Depending on the labeling strategy, inter- and intramolecular distances are accessible. Intramolecular distance constraints typically exploit doubly spinlabeled molecules, and intermolecular distances can be determined using singly labeled molecules.

EPR distance measurements rely on the dipole-dipole coupling between spins, which is inversely proportional to the 
cube of the distance. ${ }^{[95]}$ The dipole-dipole coupling also depends on the angle between the spin-spin vector and the magnetic field. Fast reorientation of the spin-spin vector, for example, fast rotational diffusion of the doubly labeled protein results in averaging over all possible orientations reducing the dipole-dipole interaction to zero. Therefore, distance measurements are most often performed in the frozen state upon shock freezing in a glass-forming solution, for example, aqueous buffer solution mixed with glycerol, resulting in an isotropic orientation distribution.

Data analysis is based either on a model of the distance distribution $^{[31,96-97]}$ or on model-free methods, for example, Tikhonov regularization. ${ }^{[90,92]}$ It is important to note that the distance between the spin density on the nitroxides differs from the corresponding distances of the protein backbone, since distance measurements are made using spin-labels such as MTSL, which possess a number of single bonds in their linkers, allowing for different rotamers (Scheme 1) which are not conformationally unambiguous. ${ }^{[98]}$ This introduces an uncertainty in the backbone-spin distance and complicates the interpretation of the spin-spin distances in terms of the protein backbone, ${ }^{[99]}$ although the uncertainty becomes less important for longer distances between the labeled sites. ${ }^{[98]}$ This uncertainty can be improved by molecular modeling of the spin label behavior. $^{[99-101]}$

Since the Zeeman interaction and, in particular, the hyperfine interaction of nitroxides are anisotropic, the EPR signal is sensitive to the molecular orientation of the label with respect to the external magnetic field. Thus, rotational diffusion can be detected by EPR, and SDSL-EPR is sensitive to dynamics on the picosecond to nanosecond timescales, covering a variety of the important molecular mechanisms in biology such as the dynamics of proteins in solution. ${ }^{[102-104]}$ The spectra do not directly report on the dynamics of the labeled macromolecule as a whole but contain information on 1) internal motion of the nitroxide about the chemical bonds of the linker (cf. Scheme 1), 2) motion of the site of attachment relative to the rest of the macromolecule (conformational flexibility), and 3) motion of the macromolecule as a whole. The internal motion of the label may be restricted by the environment, depending on the extent to which the molecular environment engulfs the label. Thus different categories of label positions, namely sites in loops or unfolded regions, sites on the surface of ordered structures, for example, helices, or sites that are buried inside the core of a protein can be identified. ${ }^{[64,105-108]}$ The periodic dependence of mobility along a sequence can be used to identify secondary structure elements and protein topography. ${ }^{[109]}$ Spin-labeled ASYN in solution at room temperature gives rise to sharp EPR lines that are characteristic for loop or unfolded regions indicating that ASYN is intrinsically disordered, that is, largely unfolded in solution.

Secondary structure information can also be obtained by studying the accessibility of the nitroxide label to paramagnetic colliders. The collision rate with the spin label influences the relaxation time of the latter, which can be measured and used to estimate the local concentration of a paramagnetic quencher near a nitroxide spin label..$^{[110-111]}$ As a consequence, by measuring the respective local concentrations, membraneburied sites can be distinguished from water-exposed sites.

\section{Alpha-Synuclein Conformations and Conformational Dynamics}

\section{Fluorescence and FRET studies}

Ensemble and single-molecule fluorescence and FRET approaches have been fruitfully used to explore structural details of ASYN conformations. Lee et al. used time-resolved Trp fluorescence energy transfer to resolve distances between donor and acceptor pairs in ASYN at physiological and acidic $\mathrm{pH}$, and in the presence of SDS micelles. FRET-derived distance distributions revealed compact, intermediate and extended conformations. The A30P disease mutant was shown to modulate the average donor-acceptor distance for a specific pair. ${ }^{[54]}$ Grupi and Haas have performed detailed time-resolved FRET experiments to explore segmental conformational disorder and dynamics in a set of chain segments from the N-terminal and core domains of ASYN to yield insights into possible initiation sites for amyloid formation. ${ }^{[60]}$ These authors have also studied the effect of temperature on segmental end-to-end distances, and observed subtle conformational biases that may play an essential role in directing the polypeptide towards productive folding or misfolding. ${ }^{[5]}$ Trp fluorescence and FRET have also been used to follow the kinetics of oligomerization and to characterize oligomeric structures of ASYN.$^{[50-51,57]}$ A systematic study of ASYN oligomers containing Trp residues engineered at positions 4, 39, 69, and 90 exhibit blue-shifted Trp spectra (relative to the monomeric protein), suggesting that these residues are well shielded from the solvent, and form the core of the oligomer. In contrast, C-terminal Trp residues (positions 124 and 140) continue to exhibit red-shifted fluorescence in the oligomeric state, indicating that the $\mathrm{C}$ terminus of the constituent monomers remains solvent exposed. ${ }^{[57]}$

A range of single-molecule FRET (smFRET) studies has also yielded insights into distance distributions in ASYN as a function of various physicochemical parameters and the presence of binding partners such as SDS micelles or large unilamellar vesicles. ${ }^{[34,68-71,112]}$ Deniz and co-workers have probed coupled binding and folding of ASYN, demonstrating a complex energy landscape on which binding of ASYN to SDS or small molecules can drive the switch from random coil to $\alpha$-helical structures. ${ }^{[68]}$ Independently, Veldhuis et al. demonstrated an all-ornone transition from an intrinsically disordered to a horseshoe shaped helical structure, yielding a higher FRET efficiency, upon binding to SDS micelles (see Figure 1). ${ }^{[34]}$

Trexler and Rhoades used smFRET to confirm the formation of a bent-helix when ASYN is bound to highly curved detergent micelles, but observed an elongated helix conformation when it is bound to large unilamellar vesicles. ${ }^{[112]}$ EPR studies have shown the coexistence of both horseshoe and elongated helix conformations on membranes (see below). Ferreon et al. have demonstrated the modulation of the folding landscape for ASYN by disease related mutations. ${ }^{[69]}$ In a recent report, Sevcsik et al. have demonstrated that oxidative modifications 

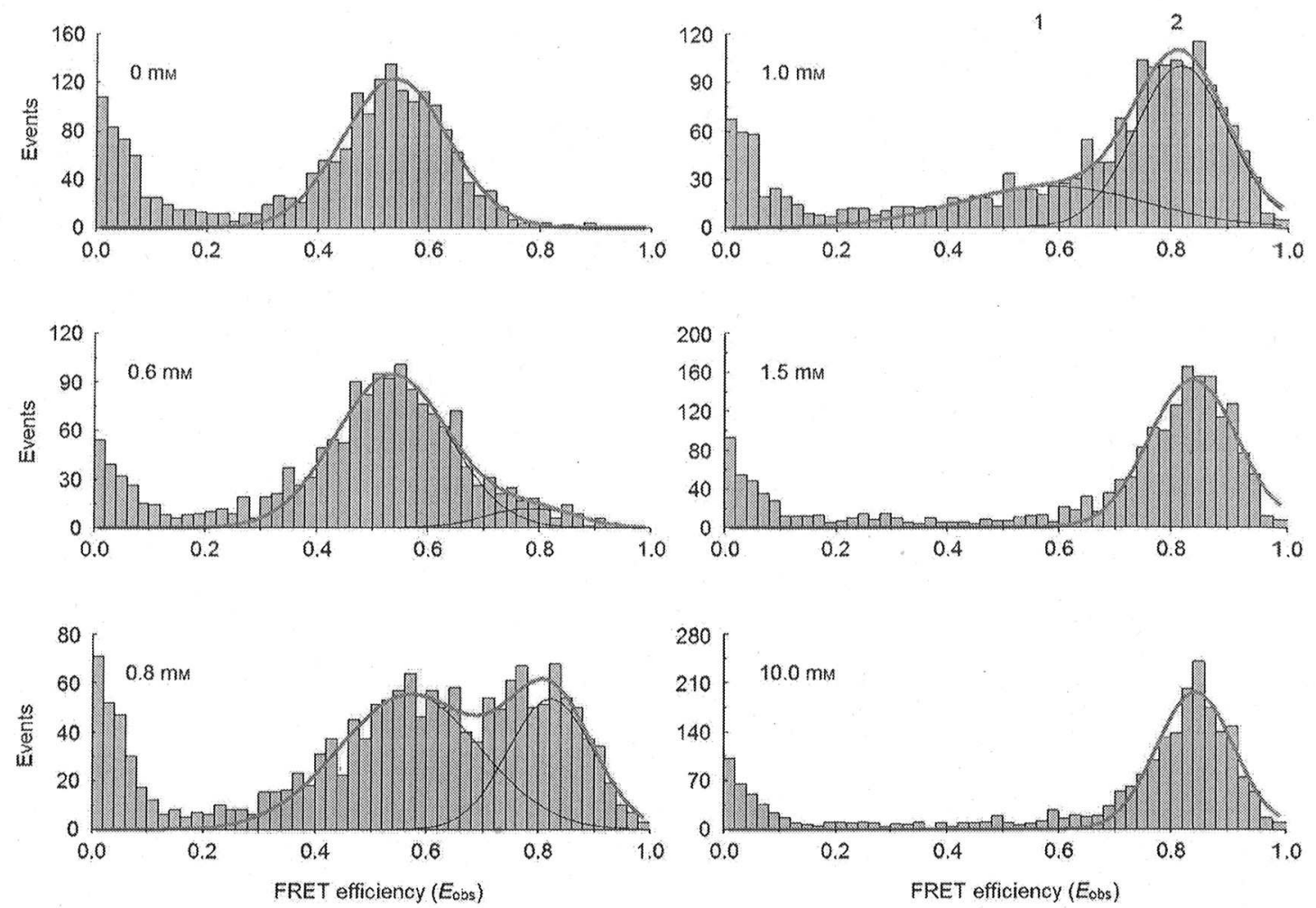

Figure 1. FRET efficiency histograms of 100 pм Alexa Fluor 488 and Alexa Fluor 568 labeled ASYN 9C/69C as a function of SDS concentration. Solid lines indi cate Gaussian fits to one or two populations (marked 1 and 2). Without SDS, ASYN conformations yield $E_{\text {obs }}$ centered at 0.54 (first panel, 0 mm). No changes in the histograms were observed up to $0.5 \mathrm{~mm}$. Upon further increasing the SDS concentration from 0.5 to $1.0 \mathrm{~mm}$, a clear second distribution centered at $E_{\mathrm{obs}} 0.82$ appeared (peak 2), indicative of a population of proteins where positions 9 and 69 are closer together, leading to increased FRET. At higher SDS concentrations (1.5 $10.0 \mathrm{~mm}$ ), the first distribution completely disappeared. Adapted from ref. [34]. Copyright: Wiley VCH, 2011.

to the $C$ terminus of ASYN influence the membrane binding ability of ASYN (modulated by the $\mathrm{N}$ terminus) by allosteric coupling, that is, by in effect depopulating those conformations capable of membrane binding. ${ }^{[113]}$

\section{EPR studies}

Upon aggregation: SDSL-EPR was used to investigate the structural features of ASYN fibrils. Fibrils grown from spin-labeled ASYN had a morphology that was similar to fibrils made from wild-type ASYN as verified by electron microscopy. Additionally, co-mixing of wild-type and labeled ASYN indicated that both species are able to adopt similar structures within the fibril, confirming that the introduction of a spin label is tolerated remarkably well. ${ }^{[14]}$ By analysing the intermolecular spinspin interaction within the fibrils in terms of dipolar broadening depending on diamagnetic dilution, it was shown that the same sites of different molecules come into close proximity. While the accuracy of this analysis was not sufficient to fully determine whether strands or sheets were parallel, ${ }^{[115]}$ a highly ordered and specifically folded central core region of about 70 amino acids was identified. The $\mathrm{N}$ terminus is structurally more heterogeneous while the 40 -amino-acid $\mathrm{C}$ terminus was completely unfolded. ${ }^{[116]}$
The latter encouraged encouraged Chen et al. ${ }^{[115]}$ to study a C-terminal truncation mutant (residues 1-115) of ASYN. This allowed for optimizing spectral quality and minimizing components from nonfibrillized protein or other background labeling due to codon mistranslation. ${ }^{[117]}$ Exchange-narrowed EPR spectra were observed for the majority of sites within the core region of ASYN fibrils. Such exchange narrowing implies orbital overlap between multiple spin labels in close contact and confirmed that the ASYN fibril core is arranged in a parallel, in-register structure wherein the same residues from different molecules are stacked on top of each other. This $\beta$-sheet-rich core region extends from residues 36 to 98 and is tightly packed.

A recent DEER study ${ }^{[118]}$ used a set of four double mutants and ten to 20-fold diamagnetic dilution to probe the distance between the extremal $\beta$-strands. The distance of $4.5 \mathrm{~nm}$ is in good agreement with the dimensions of a protofilament and suggests that three other $\beta$-sheets could lie in a parallel stack between the two extremal ones. A further study probing additional sites yielded a proposed model for three of the strands. ${ }^{[119]}$

Membrane-bound conformations: In addition to misfolding and fibril formation of ASYN, membrane binding is of particular interest for unraveling ASYN's physiological role. The $\mathrm{N}$ terminus of ASYN contains seven repeats, each of which is made up of eleven amino acids. Sequence analysis suggested that 
this region is likely to mediate lipid interactions. ${ }^{[120-121]}$ To characterize the structural changes induced by membrane binding, the EPR spectra of 47 singly spin-labeled ASYN derivatives were recorded in solution and upon binding to small unilamellar vesicles. ${ }^{[107,116]}$

As mentioned above, spin-labeled monomeric ASYN in solution gives rise to sharp EPR lines characteristic for fast rotation. Upon membrane binding, spectral changes were observed for ASYN derivatives labeled within the repeat region (residues 990). In contrast, little or no changes were detected for labels placed in the C-terminal 40 amino acids, confirming that conformational changes upon membrane binding do not occur within the C-terminal part of the protein.

Membrane-bound ASYN labeled within the repeat region exhibited line shapes indicating lipid- or solvent-exposed helix surface sites. Additionally, $\mathrm{O}_{2}$ and NiEDDA accessibilities $\left(\Pi_{2}\right.$ and INIEDDA, respectively) were determined for the labels in the repeat region. Nonpolar $\mathrm{O}_{2}$ preferentially partitions into the membrane whereas the more polar NiEDDA preferentially partitions into the aqueous phase. As a consequence, membrane-buried sites show enhanced accessibility to $\mathrm{O}_{2}$, whereas water-exposed sites are preferentially accessible to NiEDDA. $\Pi_{2}$ and $\Pi$ NiEDDA exhibit continuous periodic oscillations corresponding to a helical structure featuring the periodicity of 3.67 expected for a $3_{11}$ helix. The accessibility data for both colliders can be conveniently summarized by the depth parameter $\Phi\left[\Phi=\ln \left(\Pi_{2} / \Pi N i E D D A\right)\right]$ (Figure 2), which increases linearly with increasing immersion depth. ${ }^{[122]}$

NMR studies are limited by the size of the complex under investigation and therefore, NMR studies have been limited to the interaction of ASYN with micelles. ${ }^{[25,123-125]}$ The NMR structure of ASYN bound to SDS micelles, commonly used as a membrane mimic, revealed a break in the helix resulting in two antiparallel $\alpha$-helices. ${ }^{[25]}$ This model was confirmed by distance measurements exploiting SDSL EPR utilizing 13 different

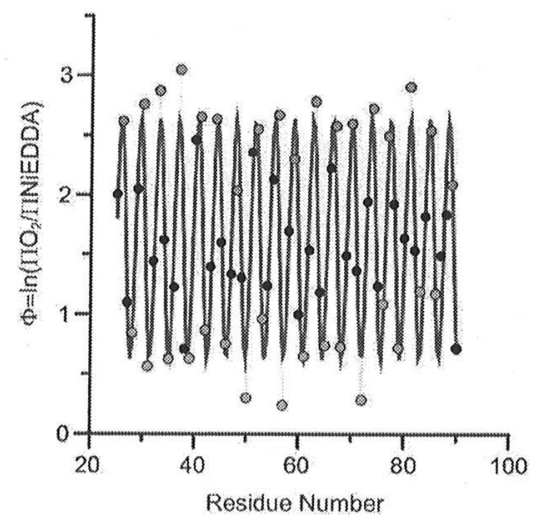

Figure 2. Solvent accessibility analysis of singly spin labeled ASYN deriva tives. The ratios of the accessibilities to $\mathrm{O}_{2}$ and NiEDDA (ПO2 and ПNiEDDA, respectively) for residues 2590 summarized by the depth parameter $\Phi=$ In((ПО2/П NiEDDA), with increasing $\Phi$ values indicating deeper membrane immersion depth. The blue line indicates the best fit to a cosine function and the resulting periodicity corresponds to the theoretically predicted pe riodicity of 3.67 amino acids per turn. Reproduced from ref. [122] with per mission. Copyright: National Academy of Sciences, USA, 2005.

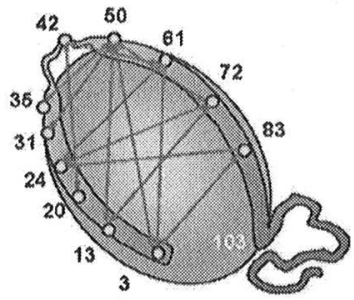

Figure 3. Cartoon representation of the two helices and linker region of ASYN bound to an ellipsoidal micelle, illustrating the different distances measured using pulsed EPR. Taken from ref. [27] with permission. Copyright: American Chemical Society, 2006.

ASYN double mutants each containing two spin-labeled cysteines (horseshoe model, Figure 3). ${ }^{[27]}$ In this study, one mutant includes a pair of cysteines placed within a single helix to provide an internal distance control. Distance distributions were obtained by DEER measurements. In studies of ASYN bound to detergent and lysophospholipid micelles, it was shown that the interhelical separation between the two helices is dependent on micelle composition. Micelles formed from longer acyl chains lead to an increased splaying of the two helices. The distance constraints were in accord with the NMR data. The data suggested that the topology of ASYN is not strongly constrained by the linker region between the two helices and instead depends on the geometry of the membrane surface to which the protein is bound.

The geometry of micelles, however, differs significantly from those of biological membranes. Micelles have typical diameters of $5 \mathrm{~nm}$ and are likely too small to accommodate ASYN in the extended conformation (around $15 \mathrm{~nm}$ for an extended helix of 100 residues). It had been postulated that the small size of the micelles might have artificially constrained the protein into a horseshoe structure. Therefore, SDSL-EPR was performed with ASYN bound to phospholipid vesicles, for example, small or large unilamellar vesicles (SUVs or LUVs, respectively).

A corresponding study considered two selected possible conformations for ASYN bound to SUVs, namely, an extended helix and the horseshoe structure. Theoretically expected spinspin distance distributions for doubly labeled ASYN taking the possible rotamers of the spin labels into account were calculated. Label positions close to the potential linker region between the two horseshoe helices were identified that would allow to distinguish between these conformations by CW-EPR distance measurements. CW-EPR spectra of labeled ASYN bound to POPC SUVs were measured and, using the theoretical distance distributions, calculated. The authors interpreted their results as ruling out an unbroken helical structure around residue 40 and confirmed the picture of the interhelix region characterized by conformational disorder. ${ }^{[126]}$ A closer inspection of these data led to the suggestion that the measured distances may be more consistent with an extended helix form than with the horseshoe model. ${ }^{[127]}$

The conformation of ASYN bound to vesicles, bicelles, and rodlike micelles was also studied by DEER, which is capable of measuring longer distances than CW-EPR. Jao et al. reported results suggesting an extended helix conformation significantly 
different from that of ASYN in the presence of SDS micelles. Their DEER study showed that for several double mutants the average distance per residue was $\pm 1 \%$ of that for an $\alpha$-helix, which argues strongly for an extended helix. ${ }^{[27.122]}$ Already in this study, a number of samples yielded somewhat bimodal distance distributions suggesting distinct conformations of the protein. This possibility was confirmed by a further DEER study, ${ }^{[128]}$ which provided direct evidence of coexisting horseshoe and extended helix conformations of membrane-bound ASYN (Figure 4).

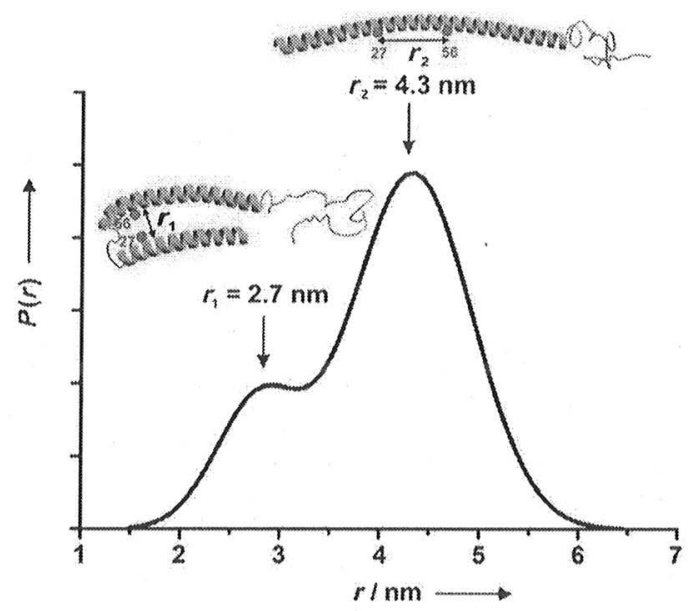

Figure 4. The distance distribution $P(r)$ for ASYN bound to LUV and spin la beled at residue 27 and 56 clearly consists of two contributions. The shorter distance agrees well with the expected distance of $2.7 \mathrm{~nm}$ for the horseshoe conformation derived from the NMR structure (PDB ID: 1XQ8) while the longer distance is consistent with an extended $\alpha$ helix. Taken from ref. [128]. Copyright: Wiley VCH, 2011.

A DEER study ${ }^{[129]}$ measuring distances of up to $8.7 \mathrm{~nm}$ showed that also the PD-linked ASYN mutations remain capable of adopting both structures, and that the protein/lipid ratio determines whether the protein adopts the broken or extended helix conformation.

The remarkable conformational flexibility of ASYN and its ability to adopt different structures is thus very likely responsible for the disparate results obtained using similar experimental techniques and often with only slight variations in experimental conditions. ${ }^{[31,122-127,130]}$

\section{Outlook}

ASYN is a model intrinsically disordered protein (IDP). IDPs comprise a large fraction of eukaryotic proteins (>30\%), and have been recognized as a unique protein class, justified by the clear structural and functional separation. They lack a welldefined three-dimensional fold and display remarkable conformational flexibility. This property potentially enables them to be promiscuous in their interactions and to adapt their structure according to the needed function. Since structure and dynamics of IDPs drastically depend on the environment, the corresponding details have been notoriously challenging to unravel.

As a result of these properties, and because of their high potential, experimental approaches exploiting site-directed labeling are amongst the most suitable methods to study IDPs and will gain increasing attention in this rapidly advancing field. These approaches have now reached a level where broad application to unraveling structure and dynamics of IDPs is feasible.

Finally, the investigation of IDPs within their natural cellular environment is crucial for understanding their physiological function and will be an important link between classical molecular biophysics and systems biology. To achieve this link, and in order to unravel the complex functions of IDPs in vivo, pushing the experimental conditions for fluorescent and EPR measurements from in vitro experiments towards structure determination in vivo pose an exciting challenge for the coming years.

\section{Acknowledgements}

We thank Marco Wassmer for preparing the TOC graphic. M.D. gratefully acknowledges funding by the Deutsche Forschungsgemeinschaft (DR 743/2-1 and CRC 969). V.S. gratefully acknowledges a TOP grant from the Chemical Sciences division of the Netherlands Organisation for Scientific Research (NWO). Research in V.S.'s laboratory is supported by NWO, the Foundation for Fundamental Research on Matter (FOM), NanoNext NL, and the Stichting Internationaal Parkinsons Fonds. Funding from NWO CW by a TOP and an ECHO grant, and from the Foundation for Fundamental Research on Matter (FOM) is gratefully acknowledged by M.H.

Keywords: alpha-synuclein - EPR spectroscopy - FRET intrinsically disordered proteins · site-directed labeling

[1] M. Goedert, Nat. Rev. Neurosci. 2001, 2, 492501.

[2] M. G. Spillantini, M. Goedert, Trends Neurosci. 1998, 21, 428433.

[3] M. G. Spillantini, M. L. Schmidt, V. M. Y. Lee, J. Q. Trojanowski, R. Jakes, M. Goedert, Nature 1997, 388, 839840

[4] C. W. Shults, J. M. Barrett, D. Fontaine, Neurosci. Lett. 2006, 405, 223 225.

[5] R. Kruger, W. Kuhn, T. Muller, D. Woitalla, M. Graeber, S. Kosel, H. Przun tek, J. T. Epplen, L. Schols, O. Riess, Nat. Genet. 1998, 18, 106108.

[6] M. H. Polymeropoulos, T. Lynch, M. Farrer, M. Hutton, J. Hardy, Science 1997, 278, 16961697.

[7] J. J. Zarranz, J. Alegre, J. C. Gomez Esteban, E. Lezcano, R. Ros, I. Am puero, L. Vidal, J. Hoenicka, O. Rodriguez, B. Atares, V. Llorens, E. G. Tortosa, T. del Ser, D. G. Munoz, J. G. de Yebenes, Ann. Neurol. 2004, 55, 164173.

[8] L. Chen, M. B. Feany, Nat. Neurosci. 2005, 8, 657663.

[9] H. Fujiwara, M. Hasegawa, N. Dohmae, A. Kawashima, E. Masliah, M. S Goldberg, J. Shen, K. Takio, T. Iwatsubo, Nat. Cell Biol. 2002, 4, 160 164.

[10] M. Okochi, J. Walter, A. Koyama, S. Nakajo, M. Baba, T. Iwatsubo, L. Meijer, P. J. Kahle, C. Haass, J. Biol. Chem. 2000, 275, 390397.

[11] J. P. Anderson, D. E. Walker, J. M. Goldstein, R. de Laat, K. Banducci, R. J. Caccavello, R. Barbour, J. P. Huang, K. Kling, M. Lee, L. Diep, P. S. Keim, X. F. Shen, T. Chataway, M. G. Schlossmacher, P. Seubert, D. Schenk, S Sinha, W. P. Gai, T. J. Chilcote, J. Biol. Chem. 2006, 281, 2973929752. [12] V. N. Uversky, J. Li, A. L. Fink, J. Biol. Chem. 2001, 276, 1073710744. 
[13] V. N. Uversky, A. Fink, FEBS Lett. 2002, 522, 913.

[14] V. N. Uversky, E. M. Cooper, K. S. Bower, J. Li, A. L. Fink, FEBS Lett. 2002 515,99103

[15] P. H. Weinreb, W. G. Zhen, A. W. Poon, K. A. Conway, P. T. Lansbury, Bio chemistry 1996, 35, 1370913715.

[16] T. Bartels, J. G. Choi, D. J. Selkoe, Nature 2011, 477, 107110.

[17] W. Wang, I. Perovic, J. Chittuluru, A. Kaganovich, L. T. T. Nguyen, J. Liao, J. R. Auclair, D. Johnson, A. Landeru, A. K. Simorellis, S. Ju, M. R. Cook son, F. J. Asturias, J. N. Agar, B. N. Webb, C. Kang, D. Ringe, G. A. Petsko, T. C. Pochapsky, Q. Q. Hoang, Proc. Natl. Acad. Sci. USA 2011, 108, 1779717802.

[18] B. Fauvet, M. K. Mbefo, M. B. Fares, C. Desobry, S. Michael, M. T. Ardah, E. Tsika, P. Coune, M. Prudent, N. Lion, D. Eliezer, D. J. Moore, B. Schneider, P. Aebischer, O. M. El Agnaf, E. Masliah, H. A. Lashuel, J. Biol. Chem. 2012; DOI: 10.1074/jbc.M111.318949.

[19] V. N. Uversky, J. Biomol. Struct. Dyn. 2003, 21, 211234.

[20] M. R. Cookson in Annual Review of Biochemistry Vol. 74, 2005, pp. 29 52.

[21] G. K. Tofaris, M. G. Spillantini, Mov. Disord. 2005, 20, S37 S44.

[22] N. M. Bonini, B. I. Glasson, Cell 2005, 123, 359361.

[23] S. Chandra, G. Gallardo, R. Fernandez Chacon, O. M. Schluter, T. C. Sudhof, Cell 2005, 123, 383396.

[24] C. B. Lucking, A. Brice, Cell. Mol. Life Sci. 2000, 57, 18941908.

[25] T. S. Ulmer, A. Bax, N. B. Cole, R. L. Nussbaum, J. Biol. Chem. 2005, 280, 95959603.

[26] E. A. Waxman, B. I. Giasson, Biochim. Biophys. Acta Mol. Basis Dis. 2009, $1792,616624$.

[27] P. Borbat, T. F. Ramlall, J. H. Freed, D. Eliezer, J. Am. Chem. Soc. 2006 128, 1000410005.

[28] R. Bussell, D. Eliezer, J. Biol. Chem. 2001, 276, 4599646003.

[29] R. Bussell, D. Eliezer, Biochemistry 2004, 43, 48104818.

[30] M. Drescher, F. Godschalk, G. Veldhuis, B. D. van Rooijen, V. Subrama niam, M. Huber, ChemBioChem 2008, 9, 24112416.

[31] M. Drescher, G. Veldhuis, B. D. van Rooijen, S. Milikisyants, V. Subrama niam, M. Huber, J. Am. Chem. Soc. 2008, 130, 77967797.

[32] D. Eliezer, E. Kutluay, R. Bussell, G. Browne, J. Mol. Biol. 2001, 307, 10611073.

[33] Y. H. Sung, D. Eliezer, J. Mol. Biol. 2007, 372, 689707.

[34] G. Veldhuis, I. Segers Nolten, E. Ferlemann, V. Subramaniam, ChemBio Chem 2009, 10, 436439.

[35] A. C. M. Ferreon, Y. Gambin, E. A. Lemke, A. A. Deniz, Proc. Natl. Acad. Sci. USA 2009, 106, 56455650.

[36] B. Caughey, P. T. Lansbury, Annu. Rev. Neurosci. 2003, 26, 267298.

[37] N. Arispe, M. Doh, O. Simakova, B. Kurganov, A. De Maio, FASEB J. 2004, 18, 16361645.

[38] N. Arispe, H. B. Pollard, E. Rojas, Mol. Cell. Biochem. 1994, 140, 119 125.

[39] H. A. Lashuel, B. M. Petre, J. Wall, M. Simon, R. J. Nowak, T. Walz, P. T. Lansbury, J. Mol. Biol. 2002, 322, 10891102.

[40] A. Quist, L. Doudevski, H. Lin, R. Azimova, D. Ng, B. Frangione, B. Kagan, J. Ghiso, R. Lal, Proc. Natl. Acad. Sci. USA 2005, 102, 10427 10432.

[41] H. A. Lashuel, B. Aljabari, E. M. Sigurdsson, C. N. Metz, L. Leng, D. J. E. Callaway, R. Bucala, Biochem. Biophys. Res. Commun. 2005, 338, 973 980.

[42] R. Kayed, A. Pensalfini, L. Margol, Y. Sokolov, F. Sarsoza, E. Head, J. Hall, C. Glabe, J. Biol. Chem. 2009, 284, 42304237

[43] Y. Yoshiike, T. Akagi, A. Takashima, Biochemistry 2007, 46, 98059812.

[44] T. Fagan, B. Kagan, L. Nault, W. Hwang, C. Glabe, R. Lal, B. Albensi, D. Teplow, Y. Sokolov, J. Alzheimer's Dis. 2006, 9, 219224.

[45] T. T. Ding, S. J. Lee, J. C. Rochet, P. T. Lansbury, Biochemistry 2002, 41, 1020910217.

[46] D. L. Pountney, N. H. Voelcker, W. P. Gai, Neurotoxic. Res. 2005, 7, 59 67.

[47] M. J. Volles, P. T. Lansbury, Biochemistry 2002, 41, 45954602.

[48] M. J. Volles, P. T. Lansbury, Biochemistry 2003, 42, 78717878.

[49] L. Breydo, J. W. Wu, V. N. Uversky, Biochim. Biophys. Acta Mol. Basis Dis. 2012, 1822, 261285.

[50] A. Dusa, J. Kaylor, S. Edridge, N. Bodner, D. P. Hong, A. L. Fink, Biochem istry 2006, 45, 27522760.
[51] J. Kaylor, N. Bodner, S. Edridge, G. Yamin, D. P. Hong, A. L. Fink, J. Mol. Biol. 2005, 353, 357372.

[52] J. C. Lee, H. B. Gray, J. R. Winkler, J. Am. Chem. Soc. 2005, 127, 16388 16389

[53] J. C. Lee, H. B. Gray, J. R. Winkler, J. Am. Chem. Soc. 2008, 130, 6898 6899.

[54] J. C. Lee, R. Langen, P. A. Hummel, H. B. Gray, J. R. Winkler, Proc. Natl. Acad. Sci. USA 2004, 101, 1646616471.

[55] H. R. Lucas, J. C. Lee, J. linorg. Biochem. 2010, 104, 245249.

[56] C. M. Pfefferkorn, J. C. Lee, J. Phys. Chem. B 2010, 114, 46154622.

[57] B. D. van Rooijen, K. A. van Leijenhorst Groener, M. M. Claessens, V. Subramaniam, J. Mol. Biol. 2009, 394, 826833.

[58] G. R. Winkler, S. B. Harkins, J. C. Lee, H. B. Gray, J. Phys. Chem. B 2006, $110,70587061$.

[59] A. Grupi, E. Haas, J. Mol. Biol. 2011, 411, 234247.

[60] A. Grupi, E. Haas, J. Mol. Biol. 2011, 405, 12671283.

[61] G. I. Likhtenshtein, J. Yamauchi, S. Nakatsuji, A. I. Smirnov, R. Tamura, Nitroxides: Applications in Chemistry, Biomedicine, and Materials Science, Wiley VCH, Weinheim, 2008.

[62] L. J. Berliner, J. Reuben, Biological Magnetic Resonance, Vol. 8, Academic Press, New York, 1989.

[63] W. L. Hubbell, D. S. Cafiso, C. Altenbach, Nat. Struct. Biol. 2000, 7, 735 739.

[64] H. S. Mchourab, M. A. Lietzow, K. Hideg, W. L. Hubbell, Biochemistry 1996, 35, 76927704.

[65] R. S. Alexander, S. K. Nair, D. W. Christianson, Biochemistry 1991, 30, 1106411072.

[66] P. G. Wu, L. Brand, Anal. Biochem. 1994, 218, 113.

[67] E. A. Jares Erijman, T. M. Jovin, Nat. Biotechnol. 2003, 21, 13871395.

[68] A. C. Ferreon, Y. Gambin, E. A. Lemke, A. A. Deniz, Proc. Natl. Acad. Sci. USA 2009, 106, 56455650.

[69] A. C. Ferreon, C. R. Moran, J. C. Ferreon, A. A. Deniz, Angew. Chem. 2010, 122, 3547 3550; Angew. Chem. Int. Ed. 2010, 49, 34693472.

[70] A. J. Trexler, E. Rhoades, Biophys. J. 2010, 99, 30483055.

[71] V. Vandelinder, A. C. Ferreon, Y. Gambin, A. A. Deniz, A. Groisman, Anal. Chem. 2009, 81, 69296935.

[72] G. Jeschke, A. Bender, H. Paulsen, H. Zimmermann, A. Godt, J. Magn. Reson. 2004, 169, 112.

[73] G. Jeschke, R. J.M. Abbott, S.M. Lea, C.R. Timmel, J.E. Banham, Angew. Chem. 2006, 118, 1074 1077; Angew. Chem. Int. Ed. 2006, 45, 10581061.

[74] G. Jeschke, ChemPhysChem 2002, 3, 927932.

[75] W. L. Hubbell, C. Altenbach, Curr. Opin. Struct. Biol. 1994, 4, 566573.

[76] K. L. Constantine, Biophys. J. 2001, 81, 12751284.

[77] G. Jeschke, Y. Polyhach, Phys. Chem. Chem. Phys. 2007, 9, 18951910.

[78] H. J. Steinhoff, B. Suess, Methods 2003, 29, 188195.

[79] M. D. Rabenstein, Y. K. Shin, Proc. Natl. Acad. Sci. USA 1995, 92, 8239 8243.

[80] A. Godt, M. Schulte, H. Zimmermann, G. Jeschke, Angew. Chem. 2006, 118, 7722 7726; Angew. Chem. Int. Ed. 2006, 45, 75607564.

[81] P. P. Borbat, J. H. Davis, S. E. Butcher, J. H. Freed, J. Am. Chem. Soc 2004, 126, 77467747

[82] L. J. Berliner, S. S. Eaton, G. R. Eaton, Biological Magnetic Resonance, Vol. 19, Academic Press, New York, 2002.

[83] E. Bordignon in EPR Spectroscopy: Applications in Chemistry and Biol ogy, Vol. 321 (Eds.: M. Drescher, G. Jeschke), Springer, Berlin, 2012 p. 121158.

[84] M. Drescher in EPR Spectroscopy: Applications in Chemistry and Biology, Vol. 321 (Eds.: M. Drescher, G. Jeschke), Springer, Berlin, 2012, p. 91 120.

[85] A. Schweiger, G. Jeschke, Principles of Pulse Electron Paramagnetic Res onance, Oxford University Press, Oxford, 2005.

[86] R. E. Martin, M. Pannier, F. Diederich, V. Gramlich, M. Hubrich, H. W. Spiess, Angew. Chem. 1998, 110, 2993 2998; Angew. Chem. Int. Ed. 1998, 37, 28332837.

[87] M. Pannier, S. Veit, A. Godt, G. Jeschke, H. W. Spiess, J. Magn. Reson 2000, 142, 331340.

[88] G. Jeschke, A. Koch, U. Jonas, A. Godt, J. Magn. Reson. 2002, 155, 72 82.

[89] M. K. Bowman, A. G. Maryasov, N. Kim, V. J. DeRose, Appl. Magn. Reson. $2004,26,2339$ 
[90] G. Jeschke, G. Panek, A. Godt, A. Bender, H. Paulsen, Appl. Magn. Reson. 2004, 26, 223244.

[91] A. D. Milov, Y. D. Tsvetkov, F. Formaggio, S. Oancea, C. Toniolo, J. Raap, Phys. Chem. Chem. Phys. 2004, 6, 35963603.

[92] Y. W. Chiang, P. P. Borbat, J. H. Freed, J. Magn. Reson. 2005, 172, 279 295.

[93] Y. W. Chiang, P. P. Borbat, J. H. Freed, J. Magn. Reson. 2005, 177, 184 196.

[94] G. Jeschke, V. Chechik, P. Ionita, A. Godt, H. Zimmermann, J. Banham, C. R. Timmel, D. Hilger, H. Jung, Appl. Magn. Reson. 2006, 30, 473498.

[95] G. Jeschke, H.W. Spiess in Novel NMR and EPR Techniques, Vol. 684 (Eds.: J. Dolinšek, M. Vilfan, S. Žumer), Springer, Berlin, 2006.

[96] S. Domingo Köhler, M. Spitzbarth, K. Diederichs, T. E. Exner, M. Dresch er, J. Magn. Reson. 2011, 208, 167170.

[97] M. Pannier, M. Schops, V. Schadler, U. Wiesner, G. Jeschke, H. W. Spiess, Macromolecules 2001, 34, 55555560.

[98] M. Sajid, G. Jeschke, M. Wiebcke, A. Godt, Chem. Eur. J. 2009, 15, 1296012962.

[99] P. G. Fajer, J. Phys. Condens. Matter 2005, 17, S1459 S1469.

[100] K. Sale, C. Sar, K. A. Sharp, K. Hideg, P. G. Fajer, J. Magn. Reson. 2002, $156,104112$.

[101] P. Fajer, S. Likai, Y. S. Liu, E. Perozo, D. Budil, K. Sale, Biophys. J. 2004, 86, 191A 191A.

[102] L. Dalton, EPR and Advanced EPR Studies of Biological Systems, CRC Press, Boca Raton, 1985.

[103] L. J. Berliner, J. Reuben, Biological Magnetic Resonace, Vol. 14, Academic Press, New York, 1998.

[104] D. E. Budil, K. A. Earle, J. H. Freed, J. Phys. Chem. 1993, 97, 12941303.

[105] J. M. Isas, R. Langen, H. T. Haigler, W. L. Hubbell, Biochemistry 2002, 41, 14641473.

[106] M. Margittai, D. Fasshauer, S. Pabst, R. Jahn, R. Langen, J. Biol. Chem. 2001, 276, 1316913177.

[107] C. C. Jao, A. Der Sarkissian, J. Chen, R. Langen, Proc. Natl. Acad. Sci. USA 2004, 101, 83318336.

[108] N. Alexander, M. Bortolus, A. Al Mestarihi, H. McHaourab, J. Meilerl, Structure 2008, 16, 181195.

[109] A. Gross, L. Columbus, K. Hideg, C. Altenbach, W. L. Hubbell, Biochem istry 1999, 38, 1032410335.

[110] W. L. Hubbell, A. Gross, R. Langen, M. A. Lietzow, Curr. Opin. Struct. Biol. $1998,8,649656$.
[111] W. L. Hubbell, C. Altenbach, C. M. Hubbell, H. G. Khorana, Adv. Protein Chem. 2003, 56, 243290.

[112] A. J. Trexler, E. Rhoades, Biochemistry 2009, 48, 23042306.

[113] E. Sevcsik, A. J. Trexler, J. M. Dunn, E. Rhoades, J. Am. Chem. Soc. 2011, $133,71527158$.

[114] M. Torok, S. Milton, R. Kayed, P. Wu, T. McIntire, C. G. Glabe, R. Langen, J. Biol. Chem. 2002, 277, 4081040815.

[115] M. Chen, M. Margittai, J. Chen, R. Langen, J. Biol. Chem. 2007, 282, 2497024979.

[116] A. Der Sarkissian, C. C. Jao, J. Chen, R. Langen, J. Biol. Chem. 2003, 278, 3753037535.

[117] M. Masuda, N. Dohmae, T. Nonaka, T. Oikawa, S. I. Hisanaga, M. Goe dert, M. Hasegawa, FEBS Lett. 2006, 580, 17751779.

[118] I. Karyagina, S. Becker, K. Giller, D. Riedel, T. M. Jovin, C. Griesinger, M. Bennati, Biophys. J. 2011, 101, L1L3.

[119] M. Hashemi Shabestari, I. M. J. Segers Nolten, M. M. A. E. Claessens, B. D. van Rooijen, V. Subramaniam, M. Huber, Biophys. J. 2012; DOI: 10.1016/j.bpj.2011.11.2493.

[120] J. M. George, H. Jin, W. S. Woods, D. F. Clayton, Neuron 1995, 15, 361 372.

[121] W. S. Davidson, A. Jonas, D. F. Clayton, J. M. George, J. Biol. Chem. $1998,273,94439449$.

[122] C. C. Jao, B. G. Hegde, J. Chen, I. S. Haworth, R. Langen, Proc. Natl. Acad. Sci. USA 2008, 105, 1966619671.

[123] S. Chandra, X. C. Chen, J. Rizo, R. Jahn, T. C. Sudhof, J. Biol. Chem. 2003, 278, 1531315318.

[124] R. Bussell, D. Eliezer, J. Mol. Biol. 2003, 329, 763778.

[125] R. Bussell, T. F. Ramlall, D. Eliezer, Protein Sci. 2005, 14, 862872.

[126] M. Bortolus, F. Tombolato, I. Tessari, M. Bisaglia, S. Mammi, L. Bubacco, A. Ferrarini, A. L. Maniero, J. Am. Chem. Soc. 2008, 130, 66906691.

[127] E. R. Georgieva, T. F. Ramlall, P. P. Borbat, J. H. Freed, D. Eliezer, J. Am. Chem. Soc. 2008, 130, 1285612857.

[128] M. Robotta, P. Braun, B. van Rooijen, V. Subramaniam, M. Huber, M. Drescher, ChemPhysChem 2011, 12, 267269.

[129] E. R. Georgieva, T. F. Ramlall, P. P. Borbat, J. H. Freed, D. Eliezer, J. Biol. Chem. 2010, 285, 2826128274.

[130] A. J. Trexler, E. Rhoades, Biochemistry 2009, 48, 23042306. 